\title{
MODIFIED IMPRESSION TECHNIQUE TO OVERCOME FLABBY ANTERIOR MAXILLARY RIDGE AND SEVERE RESIDUAL MANDIBULAR RIDGE RESORPTION - A CASE REPORT
}

\section{WENNIE FRANSISCA $^{1}$, ISMET DANIAL NASUTION ${ }^{2}$ \& RICCA CHAIRUNNISA ${ }^{3}$}

${ }^{1}$ Research Scholar, Postgraduate Program in Prosthodontics, Faculty of Dentistry, Universitas Sumatera Utara, Medan, Indonesia

${ }^{2}$ Professor, Department of Prosthodontics, Faculty of Dentistry, Universitas Sumatera Utara, Medan, Indonesia

${ }^{3}$ Lecturer, Department of Prosthodontics, Faculty of Dentistry, Universitas Sumatera Utara, Medan, Indonesia

ABSTRACT
Aim : This case report was aimed to report a succeed prosthodontic treatment for flabby anterior maxillary ridge
occluded with severe residual mandibular ridge resorption by using a modification of open mouth and closed mouth
impression technique. Background: Full denture must remain retentive and stable in the oral cavity. A non-retentive
and unstable dentures will cause the formation of excessive soft tissue (flabby ridge) on maxilla and severe ridge
resoption on mandible, thus conventional impression techniques will not produce retentive and stable denture.
Modification of open mouth and closed mouth impression technique will minimize the distortion of maxillary flabby
ridge and prevent excessive pressure on the mandibular ridge. Case Description: This report presents the
modification of open mouth impression with the dual tray technique using EVA (ethyl vinyl acetate) with stump
guides on maxilla and closed mouth functional impression with rotational handle on the mandible. Conclusion:
Combination of open mouth dual tray impression technique on maxilla and closed mouth functional impression
technique on mandible will produce denture with maximum retention and stabilization. Clinical Significance: A
combination of two different modified impression technique for flabby ridge and severe resorbed ridge was a suitable
option for achieving a retentive and stable denture.
KEYWORDS: Flabby Ridge, Severe Ridge Resorption, Open Mouth Dual Tray Impression, Closed Mouth
Functional Impression

Received: May 20, 2020; Accepted: Jun 10, 2020; Published: Jun 29, 2020; Paper Id.: IJMPERDJUN2020121

\section{INTRODUCTION}

The goal of prosthodontic treatment was to restore aesthetics, comfort and function by using dentures to replace missing teeth. Retentive and stable dentures were required to obtain the desired result. ${ }^{1}$ Alveolar ridge resorption is an unavoidable physiological change and it will be accelerated by tooth extraction and aging process. ${ }^{1,2}$ The ridge resorption was chronic and irreversible, thus if the denture were not retentive and stable, it will lead to pathological resorption. ${ }^{2,3,4}$

Pathological resorption accompany with unstable denture especially on the maxilla will cause the flabby ridge because of the higher bone resorption rate than mandible. ${ }^{3,5}$ The flabby ridge was a hyperplastic soft tissue that replaces alveolar bone that undergoes rapid resorption in patients with unstable denture for ages. ${ }^{6,7,8}$ Flabby ridge will reduced the mucosa tolerance in accepting masticatory pressure and reduced denture retention and stabilization, the mechanism of this is due to pressure on moving tissues during functional movement causing flabby 
tissue displacement and loss of denture border seal. ${ }^{4,5}$ These problems will cause the difficulty while doing the conventional impression techniques because flabby tissue must not be distorted during impression, but all limiting and supporting anatomical structures must be covered. ${ }^{9}$ Standard mucocompressive impression techniques are likely to result in an unretentive and unstable denture as the denture will be constructed on a model of the flabby tissue in a distorted state. Mucostatic techniques may not make the best use of the available tissue support and movement of the denture base relative to the support tissues may be a problem. Several impression techniques were shown to overcome those problems one of those was window technique. ${ }^{1,15}$ The use of window techniques should help to overcome some of these limitations. ${ }^{2}$ Window technique was complicated, since it was difficult to do the impression accurately while still maintaining the dimensional stability of the impression material in flabby area since there was no support from the tray, thus it require an additional pick up tray in the flabby area to maintain the dimensional stability. ${ }^{10}$ Crawford et al described the use of two impression tray with different impression material and oriented inside the oral cavity, but this technique has a disadvantage which was the difficulty of the tray orientation which tends to change position during impression process.

Severe resorbed ridge often found in mandible which has long been edentulous. This was caused by slower resorption rate in mandible than maxilla. The severely resorbed mandible will cause several problems. Those problems were athropic and thin mucosa accompany with decreased muscle tone and low pain tolerance because of the poor adaptability due to decreased supporting tissue compressibility because of shortened ridge height. Shortened ridge height will also decreased the denture bearing area to receive the masticatory pressure. ${ }^{11,13,14}$ severe ridge resorption has become the prosthodontist challenges in many years. ${ }^{1,10}$ In severely resorbed ridge, the conventional impression technique will cause the excessive pressure in the supporting tissue thus will worsen the resorption and cause the overextention of the denture base. To overcome these problem, the mucodynamic impression was introduced. ${ }^{12}$ mucodynamic impression is impression techniques with minimal pressure in accordance with the ability of the patient but still obtain a maximum denture bearing area, especially in the area of retromylohyoid flange for maximum border seal. Winkler recommends the closed mouth impression technique with tissue conditioner and light body material for severe resorbed ridge impression. ${ }^{16}$ The use of closed mouth technique can overcome the weaknesses of conventional open mouth impression techniques, because the pressure when doing the impression is adjusted by the patient functional movement thus the limiting structure and muscle movement will be obtained perfectly. ${ }^{17}$ The limitation of this techniques is the difficulty to educate elderly patients to perform the functional movement instructions that are needed when doing the impression.

The purpose of this paper is to describe the combination of two impression technique with special modification of the trays to overcome those disadvantages.

\section{Case Description}

A 52 Y.O female patient came to dental hospital Universitas Sumatera Utara. She wanted a new denture since her old lower denture was broken 2 years ago \& the patient has lost all of her teeth 10 years ago, the old upper denture was loose. Intraoral examination shows that the patient was completely edentulous in maxilla and mandible. The anterior canine to canine region in maxilla was flabby and severe resorbed ridge in posterior mandible (fig. $1 \&$ fig 2). 


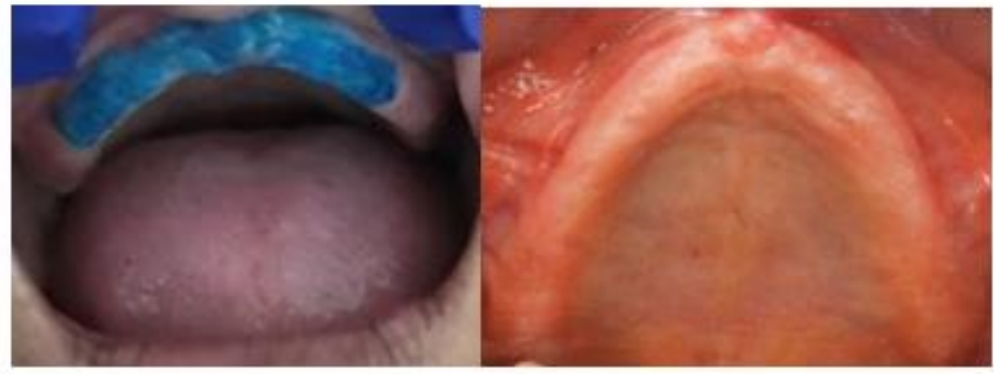

Figure 1: Full Edentulous in Maxilla with Flabby Tissue in C-C Region.

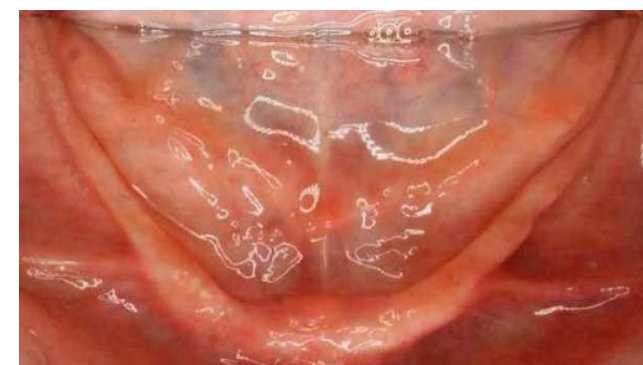

Figure 2: Full Edentulous in Mandible with Severe Resorbed Ridge in Posterior Region.

The initial treatment plan was implant supported prosthesis and surgical excision of the flabby tissue, but the patient was not willing to undergo surgical procedures so it was decided that upper and lower complete dentures will be fabricated with a different impression technique. For maxilla, the modified open mouth dual impression technique which consist of 2 trays, first was modified window tray with three stump guide (autopolymerized acrylic resin) and second was pick up tray using $2 \mathrm{~mm}$ vacum formed sheet (Ethyl vinyl acetate). For mandible, modified closed mouth impression technique with additional of rotational handle on the tray to ensure the exact position and guidance for the patient functional movement.

Preliminary impressions were taken using irreversible hydrocolloids (Hygedent, Beijing, China) with fabricated trays (fig 3) to obtainer study model and work model for custom tray fabrication (fig 4)

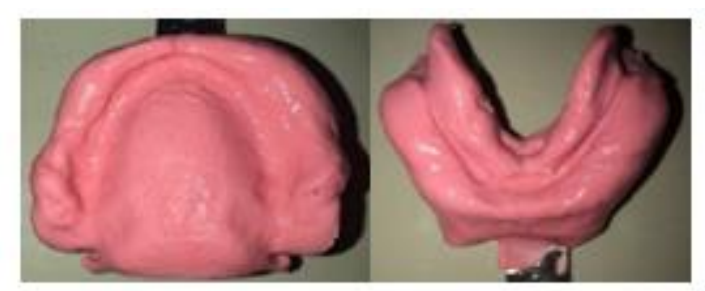

Figure 3: Preliminary Impression. 


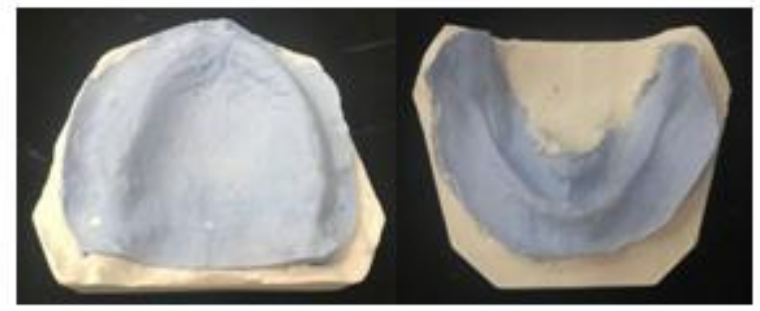

Figure 4: Study Model for Custom Tray Fabrication.

1 sheet of wax spacer was applied on the posterior region and 3 sheets of wax spacer were applied on the anterior region (CC) for flabby ridge, 2 Stoppers with kidney shape were placed in hard palate region (fig 5). Window custom tray (1,5mm) was made using a self cure acrylic resin with additional 3 stump guides (1x2mm) on left, right and middle part of the window tray (fig 6).

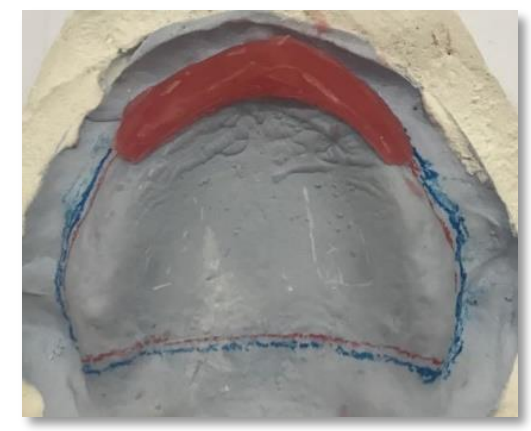

Figure 5a: Spacer for Flabby Area.

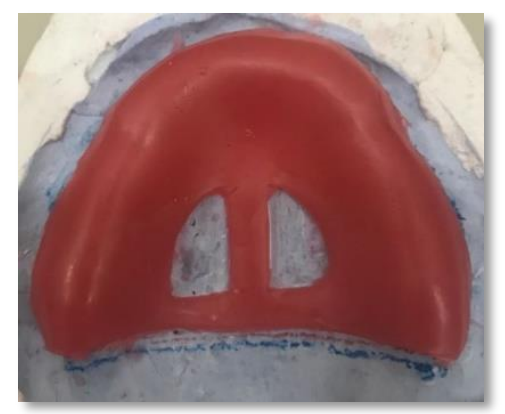

Figure 5b: Spacer and Stopper.

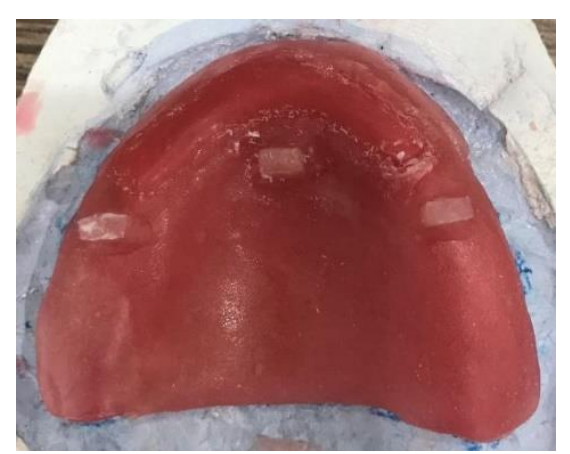

Figure 6: Modified Window Tray with 3 Additional Stump Guides

Apply plaster of paris $(1 \mathrm{~mm})$ in window area as a separator and make a pick up tray using $1,5 \mathrm{~mm}$ vacuum formed 
EVA (Ethyl vinyl acetate) and a handle with $45^{\circ}$ was made with self cure acrylic (fig 7), after that try in the custom tray inside the patient mouth and ensure the adaptation (fig 8).
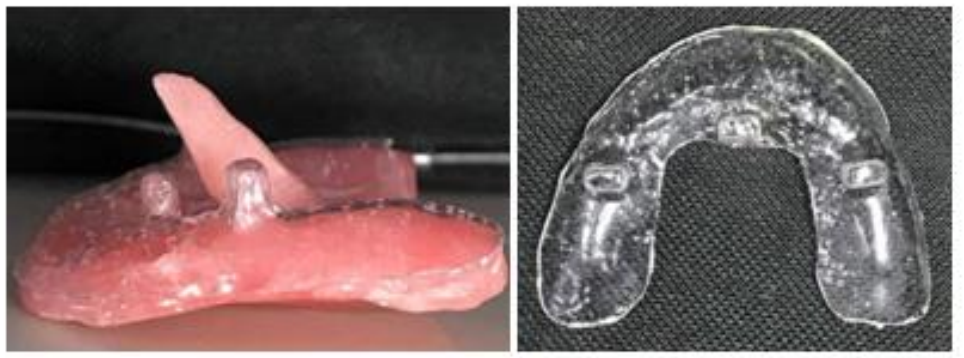

Figure 7: Pick Up Tray with EVA Material Cover the Window Tray with Sealed Movement

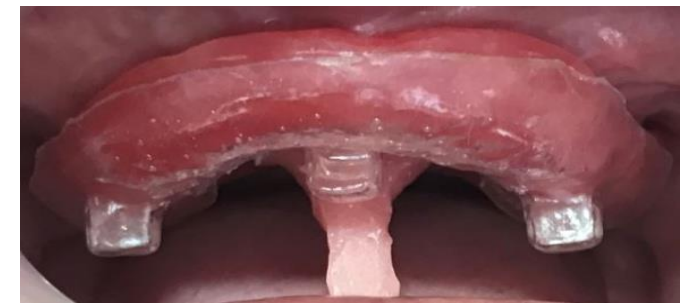

Figure 8: First Try in Adhesive Tray Material.

Adhesive Tray Material (Virtual Tray Adhesive, Ivoclar Vivadent, Germany) was applied around the border of the tray then border molding was made using Heavy body PVS material (I-Sil Premium Heavy Body, Spident Co. Ltd, Korea) Wax spacer was removed and first impression was done using regular body materal -Sil Premium Regular Body, Spident Co. Ltd, Korea) (fig 9). Several escape holes were made in pick up tray and ensure the fitting (fig 10).

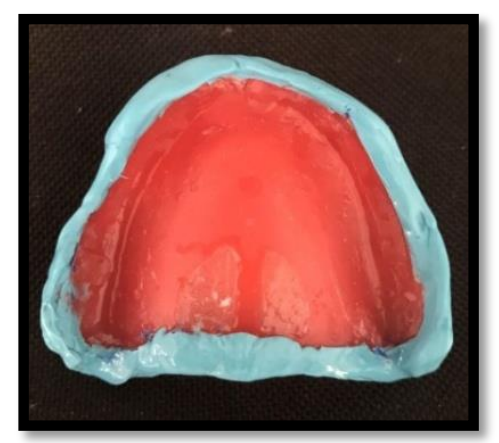

Figure 9a: Border Molding.

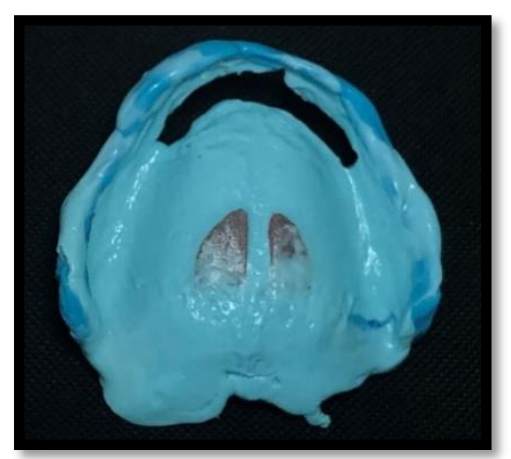

Figure 9b: First Impression Result. 


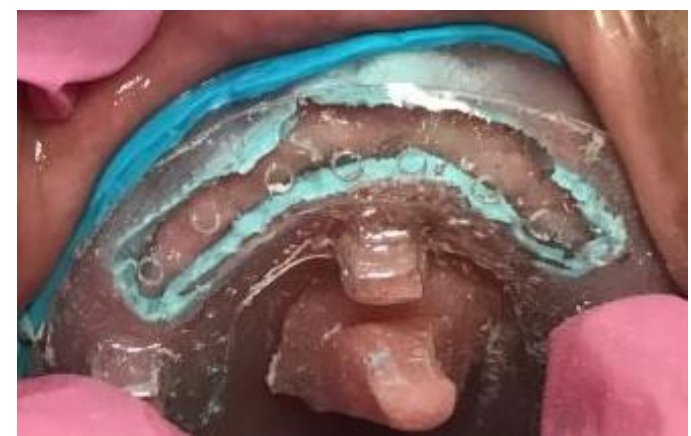

Figure 10: Second Try.

The pick up tray was removed and final impression on anterior was taken in a mucostatic situation using light body PVS, After the light body was applied, reattached the EVA pick up tray using a stump guide to ensure the position of the tray and prevent the displacement of the material. Vibrating lines, hamular notches, and flabby region were marked using indelible marker and a final cast was made using type IV stone (figure 11).

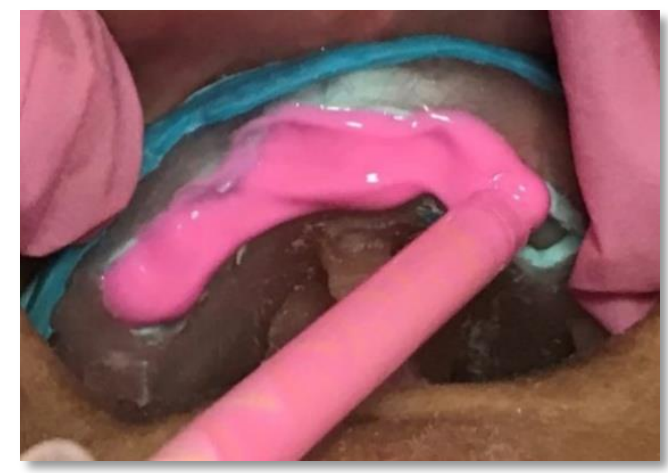

Figure 11a: Apply Light Body.

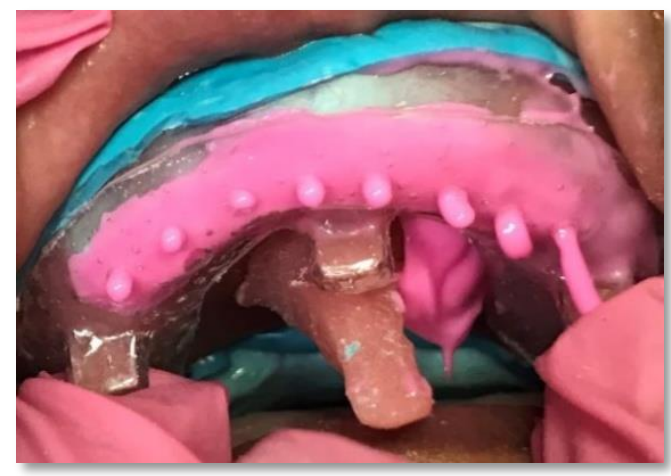

Figure 11b: Inserting an EVA Pick Up Tray.

For mandible, the custom tray was made using self-curing acrylic resin, where only one sheet of wax spacer was applied on mandibular diagnostic cast. The rotational handle was made using a bolt and hard straw as a mould with self cure acrylic resin, make sure the rotational handle did not hinder the lip movement (figure 12) 


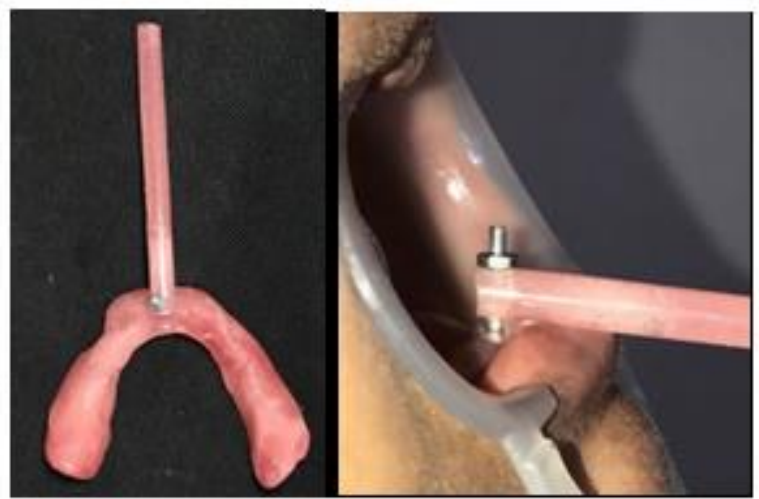

Figure 12: Custom Tray With Modified Rotational Handle.

Lower biterim was made on the custom tray following the neutral zone, then the, tray was placed inside the patient mouth and patient was told to hold the rotational handle at the center of the mouth. The patient was instructed to just keep the tray in the mouth for 5-10 min, so the patient will get familiarized and better adjusted with custom tray (figure 13).
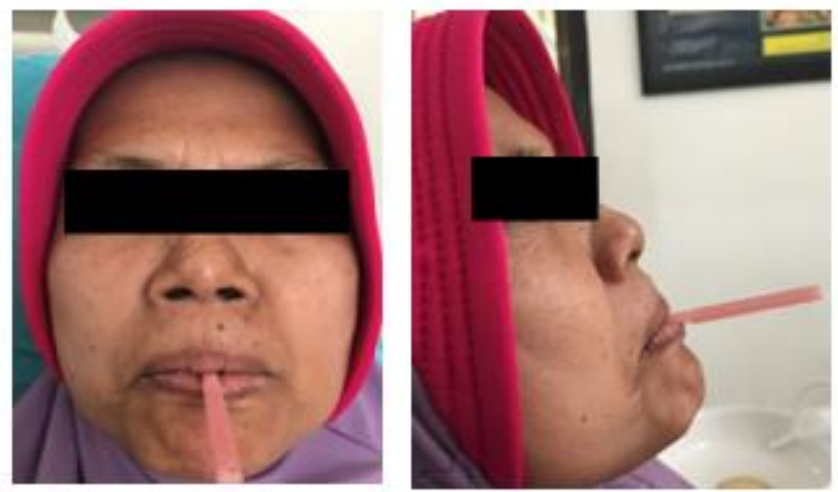

Figure 13: Adjusting the Custom Tray.

Border molding using tissue conditioner (GC Soft liner, GC Corporation Tokyo, Japan), material was carried out by placing the material on inner border of the tray. Swallowing and chewing functional movements were performed first by keeping the handle at center of the mouth. The patient was instructed to concentrate on handle while doing the functional movements to record the labial flange and anterior lingual flange. Rotational handle was moved to the left corner of the mouth, \& move slowly the right lip, right cheek, and tongue to the left corner of the mouth by concentrating on the handle to record right buccal flange and right lingual flange to retromylohyoid area. Similar procedure but opposite movements was carried out to record left buccal and lingual flange. Finally, distolingual flange was recorded by instructing to do swallowing movements bilaterally that will stimulate palatoglossus bilaterally (figure 14). 


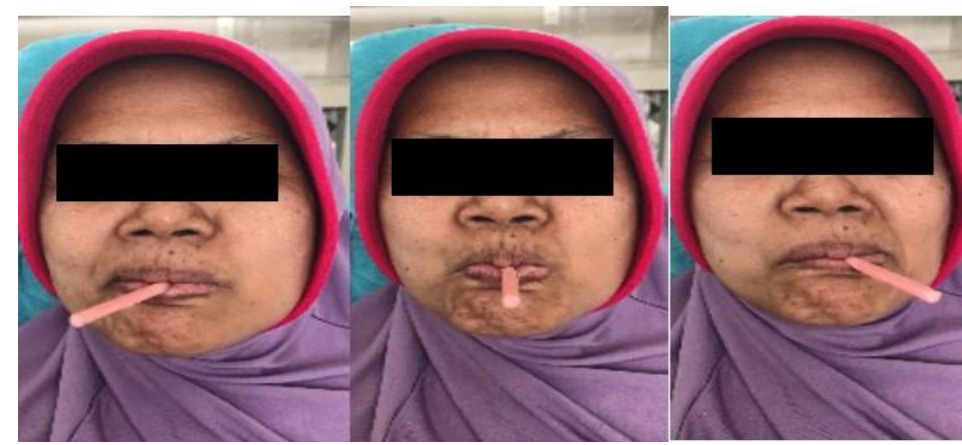

Figure 14: Functional Movement by the Patient.

After checking the border molding, the rotational handle was removed and excessive material was trimmed. Remove the wax spacer from inner side of the tray, replace the wax spacer with tissue conditioner material. The closed mouth functional impression was performed by instructing the patient to done puffing, blowing, speaking, swallowing and moved her tongue to all direction with 10 minutes interval. The final impression was checked and final cast was made using stone type IV (figure 15).
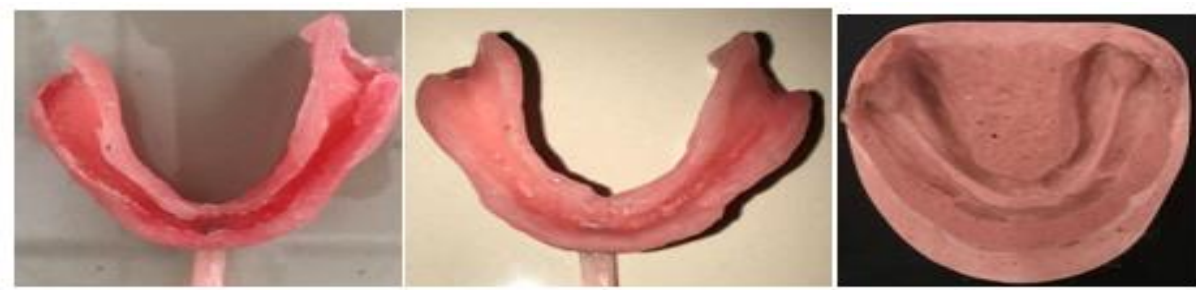

Figure 15: Border Molding Border Molding and Impression Result.

Occlusal bite rims were established and adjusted with neutral zone to obtain definitive vertical dimension. The record was transferred to semi adjustable articulator (CSA 650 Corident, Daegu, South Korea). Artificial teeth arrangement was done with lingualized occlusion with semi anatomical teeth and 0 overbite after try in was done, the denture was packed and cured. The denture was polished and inserted to the patient mouth (figure 16).
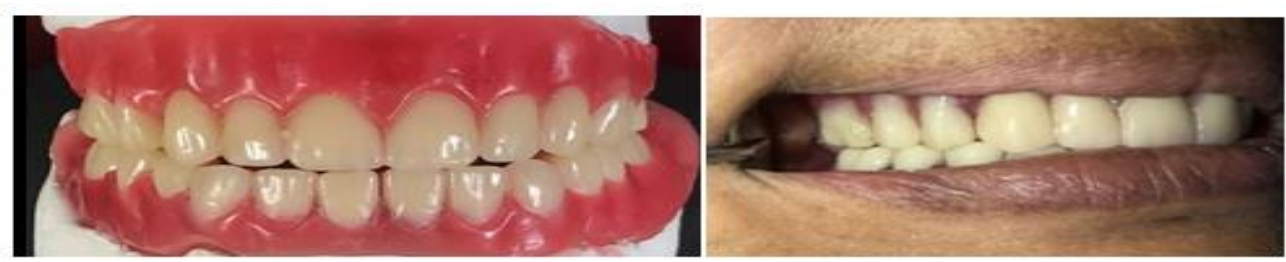

Figure 16: Artificial Teeth Arrangement and Final Insertion.

\section{DISCUSSIONS}

Retentive and stable denture fabrication are closely related to impression technique. ${ }^{1,9}$ Dentures must remain stable and retentive when the patient was doing functional movement such as talking, eating, or when coughing and sneezing, and also have a good aesthetic. ${ }^{3}$ In the case with flabby ridge on anterior maxilla combined with severe resorbed ridge on posterior mandible, conventional impression technique cannot be used because it will cause the flabby ridge distortion and loss of peripheral seal when functional movement. ${ }^{4}$

In this case, the problem with the maxilla is the flabby tissue in the anterior ridge, the main point is to prevent the flabby tissue distortion when doing the impression. Dual tray impression printing technique was done with modification to 
maintain the dimensional stability of the impression. The stump guides serve as lock for the pick up tray to prevent rotation when doing the mucostatic impression. EVA material was chosen as pick up tray material because it was easy to manipulate, good adaptation and translucent thus will provide a good vision of the impression material flow. EVA material was also rigid thus it can maintain the dimensional stability of the PVS. The use of PVS impression material is preferred by clinicians because it is available in various viscosities which can be adjusted for mucodynamic impression. The use of regular body PVS with mucocompressive pressure is done on the normal ridge. Whereas PVS light body was used for flabby area mucostatic impression which aims to minimize the pressure in the flabby area. ${ }^{5}$

The mandible problem is the severe resorbed ridge in the posterior region. The keypoint was to obtain the maximum denture bearing area without causing the excessive pressure on the residual ridge. ${ }^{10}$ All the functional movement must be recorder perfectly to prevent the loss of border seal. Closed mouth functional impression was the best choice because it provide the dynamic movement according to the patient capacity. But the disadvantage of this technique was the difficulties to control the patient movement precisely. Thus it require a special training and impression material with longer setting time to give the patient enough time to record all the functional movements. PVS material was not chosen because of the short setting time. Tissue conditioner was the material of choice because of the longer setting time (around 10 minutes). Thus it will give the patient enough time to record all the movement. Also, this material can be easily added in case there are any defects in the impression, because of the material homogeneity. The rotational handle will guide the patient movement and improve the patient motoric ability.

\section{CONCLUSIONS}

Unstable denture can alter the character, condition and form of the underlying oral tissues. The pathological changes must be carefully examined and resolved, prior to the beginning of the new prosthetic rehabilitation. A thorough history, a keen eye in clinical examinations and treatment planning will help the prosthodontist to provide his patients with satisfactory complete denture prosthesis.

\section{Clinical Significance}

A combination of two different modified impression technique for flabby ridge and severe resorbed ridge was a suitable option for achieving a retentive and stable denture.

\section{REFERENCES}

1. Zarb., et al. Prosthodontics Treatment for Edentulous Patients. 2013. $13^{\text {th }} \mathrm{Ed}$

2. Jayaprakash MB, Sahu K, Khan M, et al. Management of Flabby Ridge Cases: A Challenge in Clinical Practice. Int J Adv Health Sci 2014; 1(5):32-7.

3. Pai UY, Reddy VS, Hosi RN. A Single Step Impression Technique of Flabby Ridge Using Monophase Polyvynylsiloxane Material: A Case Report. Case Report in Dentistry 2014.

4. Labban, N. Management of the flabby ridge using a modified window technique and polyvinylsiloxane impression material. The Saudi Dental Journal 2017, 30(1): 89-93.

5. Chawara SH, Fernandes F. Tissue stress evaluation at border seal area using patient manipulated custom tray-modified closed mouth functional technique for flat mandibular ridge. JIOH, 2018, vol 10(2).

6. Prasad KP, et al. enchancing stability: a review of various occlusal schemes in complete denture prosthesis. NIJHS, 2013. Vol 
$3(2)$

7. Prasad K, et al. Prosthodontic management of compromised ridges and situations. 2014. Vol 4(2).

8. Reddy V, Srirambalaji S. A Varied Approach in Managing Fibrous Ridges. JIDENT 2017; 3(2), 1-5.

9. Khinnavar, PK, Kumar BHD, Shivakumar HR, Nandeeshwar DB. Multidisciplinary approach in rehabilitating flabby ridge in completely edentulous patient. European Journal of Prosthodontics 2015; 3(2): 51 -4.

10. Reddy VS, SriramBalaji S. A Varied Approach in Managing Fibrous Ridges. JIDENT 2017; 3(2): 1-5.

11. Prasad KD, Mehra D, Prasad AD. Prosthodontic Management of Compromised Ridges and Situations. NUJHS 2014; 4(1): 1417.

12. Arora AK, Goyal I, Sehgal M. Comparative Evaluation of Reproducibility of Pheripheral Tissues Produced by Different Border Molding Materials in Edentulous Patients: An in vivo Study. J Indian Prosthodont Soc 2015; 15(2): 102-110.

13. Drago CJ. A Retrospective Comparison of Two Definitive Impression Technique and Their Associated Postinsertion Adjustments in Complete Denture Prosthodontic. Journal of Prosthodontics 2003; 12(3): 192-7.

14. Yilmaz B, Ozcelik TB. An Alternative Impression Technique for Complete Dentures. Journal of Prosthetic Dentistry 2014; 111: $166-8$.

15. Jayaprakash, M. B., Sahu, K., Khan, M., Khoriya, S., Jadhav, S., Kendre, B. and Lukaram, A. Management offlabby ridge cases: A challenge in clinical practice. Int J Adv Health Sci 2014; 1:32-37.

16. Shanahan, T., E., J. Physiologic jaw relations and occlusion of complete dentures. J Prosthet Dent 2004; 9:203-5.

17. Shah, V., Dhaded, S. and Sajjan, C. Lingualized Occlusion: A Better Way for Enhancing Function and Esthetic. Indian J Basic and App Med Res. 2015; 5(1): 484-488.

18. Gupta, A. N. S. U. I. A., et al. "Tooth Supported Mandibular Overdenture: A Forgotten Concept." TJPRC: International Journal of Prosthetic Dentistry \& Research (TJPRC:IJPDR) 1.1 (2017): 5-10.

19. Kaur, M. A. N. E. E. T., S. U. M. I. T. Makkar, and S. Kidwai. "Anthropometric evaluation of buccal alveolar bone dimensión of maxillary anterior teeth in indian population: A cone beam computed tomography study." International Journal of Dental Research \&Development (IJDRD) 6.6 (2016): 23-32.

20. VASUDHA, D., et al. "POLYMERS AND PLASTICIZERS USED IN TRANSDERMAL DRUG DELIVERY: AN OVERVIEW." IASET: International Journal of Applied Chemistry and Pharmaceutical Sciences (IASET: IJACPS) 1.1 (2016):1-14

21. EID, S., and A. MANSOUR. "STUDY ON THE EPR/DOSIMETRIC PROPERTIES ON DL-TRYPTOPHAN." International Journal of Applied and Natural Sciences (IJANS) 3.4 (2014):29-36

22. LOHAKARE, SANDHYA SHYAM. "FAST MOVEMENT OF TEETH DURING ORTHODONTIC TREATMENT." International Journal of Dental Research \& Development (IJDRD) 4.6 (2014):7-14

23. SHANKAR, UMA, et al. "ORTHOMORPHIC SURGERY FOR MANDIBULAR DEFORMITY-A CASE SCENARIO." International Journal of Dental Research \& Development (IJDRD) 9.2 (2019):1-10

24. MAHESH, LANKA, et al. "IMMEDIATE IMPLANT PLACEMENT IN POSTERIOR MAXILLA." International Journal of Dental Research \& Development (IJDRD) 8.1 (2018):1-4 